\title{
Glove-based systems for medical applications: review of recent advancements
}

\begin{abstract}
Human hand motion analysis is attracting researchers in the areas of neuroscience, biomedical engineering, robotics, human-machines interfaces (HMI), humancomputer interaction (HCI), and artificial intelligence (AI). Among the others, the fields of medical rehabilitation and physiological assessments are suggesting high impact applications for wearable sensing systems. Glove-based systems are one of the most significant devices in assessing quantities related to hand movements. This paper provides updated survey among the main glove solutions proposed in literature for hand rehabilitation. Then, the process for designing glove-based systems is defined, by including all relevant design issues for researchers and makers. The main goal of the paper is to describe the basics of glove-based systems and to outline their potentialities and limitations. At the same time, roadmap to design and prototype the next generation of these devices is defined, according to the results of previous experiences in the scientific community.
\end{abstract}

Keywords: glove-based systems, wearable systems, smart fabrics, human body monitoring, telemedicine, $\mathrm{HMI}$
Volume 4 Issue 3 - 2018

\author{
Giorgio De Pasquale, Leonardo \\ Mastrototaro \\ Department of Mechanical and Aerospace Engineering, \\ Polytechnic University of Turin, Italy
}

Correspondence: Giorgio De Pasquale, Department of Mechanical and Aerospace Engineering, Polytechnic University of Turin, Corso Duca degli Abruzzi, 24, I0I29 Torino, Italy, Tel 0I10906912/6912, Email giorgio.depasquale@polito.it

Received: April 26, 2018 | Published: June 28, 2018
Abbreviations: HMI, human-machines interfaces; HCI, human-computer interaction; AI, artificial intelligence; DoFs, degrees of freedom; DIP, distal interphalangeal; PIP, proximal interphalangeal; MCP, metacarpophalangeal; TMCP, trapeziometacarpal; IMU, inertial measurement units; IMMS, inertial and magnetic measurement systems; EMG, electromyography; MPU, motion processing unit; MARG, magnetic, angular rate and gravity; EKF, extended kalman filters; ADC, analog-to-digital; SPI, serial peripheral interface; WPAN, wireless personal area network; USB, universal serial bus; GUI, graphical user interface; API, application program interfaces; PMU, power management unit

\section{Introduction}

The sense of touch allows humans and animals to perform coordinated and efficient interactions within their environment. Our hands drive us in a huge number of tasks in our everyday life. Then, hand function impairment caused by neurological disorders, such as stroke and cervical spinal cord injury, has negative impact on the quality of life of affected people. Physical training therapies have primary clinical relevance to improve motion recovery. Therefore, intense research efforts have been addressed to the development of upper limb rehabilitation therapies based on glove systems. In fact, instrumented gloves allow monitor hand motion during rehabilitative exercises needed to restore, totally or partially, patients' original abilities. Wearable devices should include some basic capabilities: firstly, the simultaneous recording of dynamic finger movements during the exercise (e.g. grasping objects, pinching, etc.), then the quantitative measurement of slight improvements in hands and limbs mobility. Finally, instrumented gloves should provide the continuous measurement of patients' motion all day if necessary.

This paper has the goal to provide exhaustive survey report of glove-based systems for medical applications. The high number of researches, laboratory demonstrators and prototypes published in last year's determines low level of systematic organization of data in the field of instrumented gloves applied to rehabilitation. The last available wide review summary about glove systems and their applications ${ }^{1}$ is not selectively focused on medical applications, which are the target of the present state of art analysis. This paper aims to provide research guidelines of the state of art for novices and experts operating in smart wearable systems in clinical field. The paper is organized in four sections. After the introduction to the wearable sensing topic, the state of art of the current technologies linked to instrumented gloves is presented. Here, extensive overview of most important devices is reported. The third section focuses on the main design issues, by including motivations and comparison among different architectural solutions. The key features of glove-based systems are discussed, including materials, sensor types and sensing strategies, validation tests, calibration, interfacing modules, and power management.

\section{State of the art}

Glove-based systems for medical applications and, especially, motion rehabilitation are primarily addressed to hand functional assessment. The evaluation of hand functionalities for clinical purposes requires the acquisition of multiple types of data, including for example pinching and gripping force, temperature, motion ranges of hand joints, etc. Several typologies of sensors have been used to measure the physical quantities of interest, with dedicated architectural and circuital layouts. Solutions provided by wearable devices allow reduced invasiveness, increased accuracy and repeatability, improved complexity of measurements thanks to simultaneous operability of different sensors.

Human hand contains 27 bones with roughly 25 degrees of freedom (DoFs), which are driven by 17 intrinsic muscles in the hand itself and 18 extrinsic muscles in the forearm. This complex system is capable of performing more complicated and dexterous tasks than any other existing systems. Also, it has been regarded as a rich source of inspiration for the engineers and scientists to design human-like robotic and prosthetic hands and to learn and model human hand 
motion skills. Figure 1 shows the hand joints and the DoFs typically used to describe hand motion. The distal interphalangeal (DIP) and proximal interphalangeal (PIP) joints of each finger have 1 DoF each (flexion/extension), while the metacarpophalangeal (MCP) joints have 2 DoFs (flexion/extension and abduction/adduction). The third DoF of the trapeziometacarpal (TMCP) joint allows the thumb to rotate longitudinally as it is brought into opposition with the fingers.

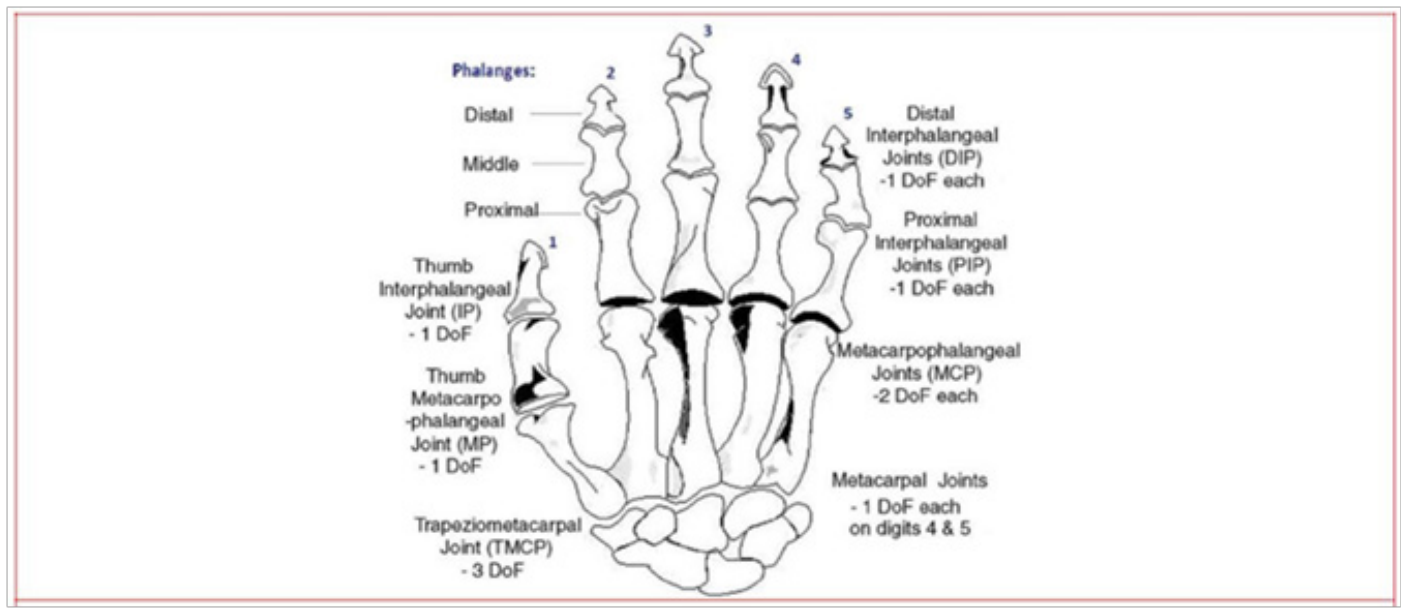

Figure I Hand fingers, joints, and related DoFs.

Arm and hand functions have been found to decline with age ${ }^{2}$ as the result of motion impairments such as decreased coordination, decreased manual dexterity and reduced grip strength. Older adults' arm and hand function also is reduced because of impairments related to common diseases in this population, such as rheumatoid arthritis and osteoarthritis, fractures and neurological conditions, such as strokes. In this last case there exist specific rehabilitation trainings that stroke survivors can perform to restore their lost abilities. Medical and engineering literature is plenty of rehabilitation tasks consisting in simple and repetitive operations to be evaluated by the trainer. A typical sequence of training exercise supported with instrumented glove may include ${ }^{3}$ :

a. To take a bottle from the table, pour water into a glass and put the bottle back on the table

b. To unscrew the lid of a jar and put the lid on the table

c. To remove a peg from the hole of a square pegboard and place it into the hole of another pegboard

For each task, the score of a patient is established by calculating the correlation between his recorded data curve and the curve of a healthy subject. Thus, quantitative characterization of hand movements is of great significance, not only for understanding pathophysiological aspects of finger neural control but also for quantifying impairments of hands, guiding appropriate therapies and assessing the effectiveness of treatments. Therefore, glove-based devices are recognized as the most promising solution to assess hand functions because of accuracy, capability of recording hand kinematics and insensitivity to subjective evaluations.

\section{Glove-based systems}

In general, glove-based system is defined as a hand-worn device including sensors array, specific electronics for data acquisition/ processing and power supply. Sensors and electronic components are sewn or printed on a fabric support that can be worn on the user's hand to record data related to hand configuration/motion. Over the years a huge number of gloves with different designs have been proposed: each glove has different functions, sensors and interfaces able to respond to specific requirements.

The first glove-based device that spreads its popularity worldwide was the Data Glove, ${ }^{4}$ presented in United States in 1987. Firstly developed by Zimmerman, ${ }^{4}$ the original version of the Data Glove used thin flexible plastic tubes sewn on a cloth and light sources and detectors to record joint angles. It was equipped with 5 to 15 bending sensors: 8 are addressed to measure MCP and PIP joints flexion on 4 fingers and 2 for the thumb. In some cases, abduction/adduction sensors are used to measure angles between adjacent fingers. In 1997, Humanglove $^{5}$ was developed by a spinoff company of the Scuola Superiore Sant'Anna (Pisa, Italy). It was designed to measure the fingers joint angles with 20 Hall-effect sensors (four sensors for each finger), as well as fingers and thumb abduction/adduction. In the same year, the goniometric SIGMA (Sheffield Instrumented Glove for Manual Assessment) Glove ${ }^{6,7}$ was commercialized. It incorporated carbon ink bend sensors in order to detect finger joints angles. In 2007, the Shadow Glove Monitor ${ }^{8}$ was presented, equipped with up to eight resistive sensors for finger bending. It can operate in three data collection modes, which are automatically selected and controlled by the arm unit software. Three models are the Sample-and-Send Mode:

i. In which the arm unit measures and transmit data immediately to storage computer by wirelessly without locale storage in the unit, the Sample-and-Save Mode

ii. Where data are collected and stored locally on the arm unit and no external computer is required, and the Sample-and-Dump Mode

iii. In which new data are sampled and transmitted with buffering of local storage memory. Modes 2 and 3 required $1 \mathrm{MB}$ local storage memory.

In 2009, one of the first glove-based systems ${ }^{9}$ able to provide a feedback to the user has been developed. This technology harvests waste mechanical energy, then used to suppress hand tremors. This kind of diseases, such as Parkinson and arthritis, are characterized 
by mechanical signals measured by piezoelectric sensors, filtered, converted and stored, or used directly to suppress the tremors. In the same year, two researchers, Gentner and Classen, developed at the German university of Würzburg the WU glove ${ }^{10}$ for hand rehabilitation. It has 14 resistive sensors to measure finger bending and ab/adduction. One of the most popular data glove systems is the CyberGlove: since 2010 the third version of this prototype, namely CyberGlove III $^{11}$ (Figure 2) has become available on the market. This glove provides maximum 22 bending sensors placed along each MCP, PIP and DIP finger joints, between each finger to detect abduction, and across the back of the hand.

Again in 2010, the low-cost instrumented glove prototype NeuroAssess Glove ${ }^{3}$ (Figure 3) has been developed for the routine assessment of hand function in clinical practice and rehabilitation settings.

The X-IST Data Glove ${ }^{12}$ has been commercialized in 2011. This glove contains either a combination of 5 bending sensors and 5 pressure sensors, or 15 off-the-shelf bending sensors, or two-axes accelerometer. Bending sensors are placed along each of the three finger joints and the thumb. Alternatively, pressure sensors are placed on each fingertip. Sensors are positioned on an inner cotton glove and protected by an outer silk glove. The low-cost bimanual rehabilitation system Atlas ${ }^{13}$ has been designed for in-home use by post stroke patients to improve hand and upper extremity function. It has four components: gloves, IMUs (inertial measurement units), resting base for hands, and electronics unit. The glove embeds potentiometer bending sensors to measure finger joint angles. Born in 2013, the Tyndall/UU Glove ${ }^{14}$ uses a combination of 20 bend sensors, 16 triaxial accelerometers and 11 force sensors to detect joint movements. All sensors are placed on a flexible PCB to provide high levels of flexibility and sensor stability. Another instrumented glove was developed by a team of Indian researchers ${ }^{15}$ which records the force exerted by the thumb, index and middle finger on an object. The multiple-sensor hand motion capture system described in ${ }^{16}$ is able to capture the finger trajectories, the contact force signals and the muscle signals. It included high-accuracy finger joint angle measurement system, i.e. the aforementioned CyberGlove, wireless tactile force measurement system from Finger TPS ${ }^{17}$ and high frequency EMG (electromyography) capture system with Trigno Wireless Sensors.
Datag loves are a wide family of sensing devices worn on the hand, which exploits different strategies to get kinematic data. The most popular sensing methods are resistive-bending sensors and optical fiber sensors, with the latter one giving the highest accuracy $(<$ $1 \mathrm{deg}$ ). In both cases, the relative orientation of articulated segments is measured by mounting the sensor across the joint of interest. However, severe limitations are due to sensor placement and needing of accurate alignment between sensors and joint. Additionally, frequent re-calibration procedures are required during utilization to mitigate the errors caused by sensors movements on the flexible substrate. To overcome this problem, the ambulatory system using inertial and magnetic sensors reported in Kortier ${ }^{18}$ has been proposed: sensors are placed on fingers and thumb and allow full 3D reconstruction of all fingers and thumb joints as well as the absolute orientation of the hand. The wearable tactile data-glove ${ }^{19}$ with 54 tactile cells was made in 2015 by using pressure sensors to measure contact forces. Here, the authors have introduced a fabric-based flexible and stretchable tactile sensor, which is capable of seamlessly covering natural shapes. The glove design allows embedding several tactile cells in a single sensor patch with arbitrary perimeter and free-form surface. The instrumented glove reported in $^{20-22}$ uses feedback technique by means of rotation measurement of each finger joint and pressure on fingertips. Angular position sensors and pressure sensors are used at this purpose. Since the majority of the rehabilitation exercises involve movement coordination, five motors and LEDs have been added to produce vibration and visual stimuli to indicate which finger has to be moved during rehabilitation routines. The glove also tracks hand rotation and translation using a motion processing unit (MPU) including one accelerometer and gyroscope. The MPU communicates via serial peripheral interface protocol with an HMI module where a graphical application ${ }^{20}$ was developed in order to monitor each finger and to implement rehabilitation game-like exercises. The sensor glove called FuncAssess Glove ${ }^{23}$ (Figure 4) was recently developed. It is based on resistive bending sensors and resistive force sensors; the first ones are placed on the dorsal sides of finger joints to monitor their bending angles, while the latter ones are situated on the fingertips to measure forces exerted to objects. The last significant case in literature is the sensing glove ${ }^{24}$ presented in 2016 that uses innovative strategy to measure fingertips contact forces with deformable objects: traditional force sensors are replaced with magnetic, angular rate and gravity sensors.

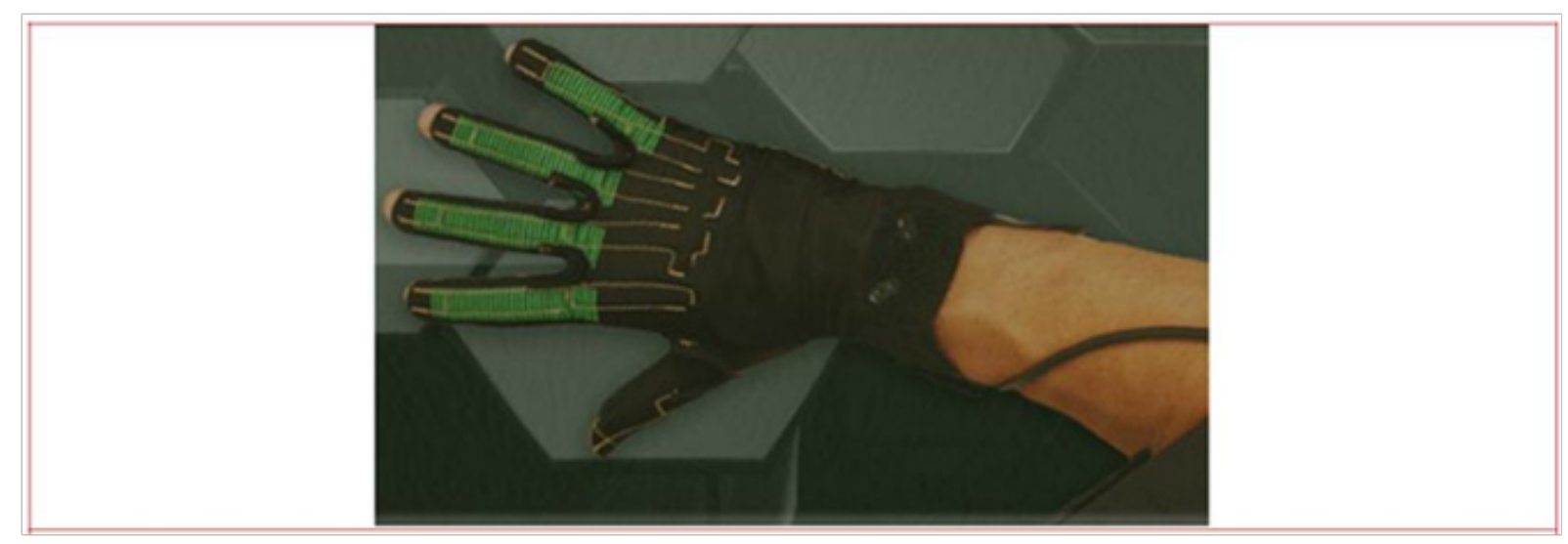

Figure 2 Cyber Glove III." 


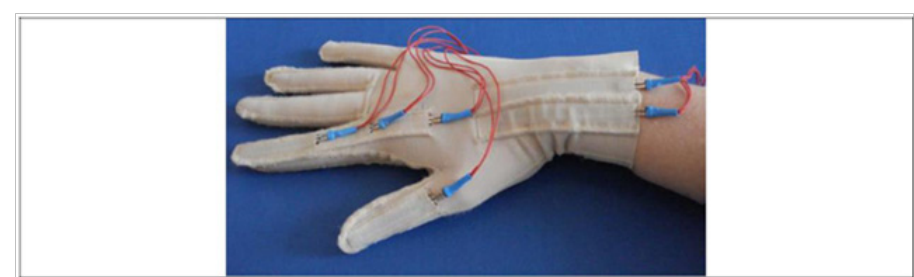

Figure 3 Top side view of the NeuroAssess Glove. ${ }^{3}$

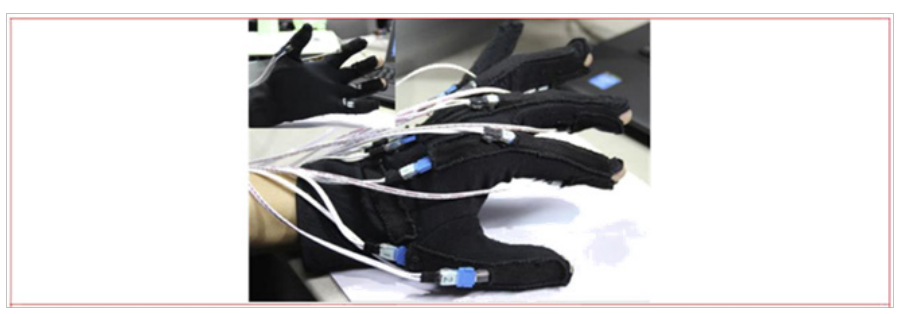

Figure 4 The Func Assess Glove. ${ }^{23}$

\section{Design issues}

The aim of this section is to analyze the detailed issues concerning the design of glove-based systems and to describe their technical features and strategies including selection of transducers and materials, electronic architectures and user interfaces. The same prototypes and devices described in Section 2 are used as references to provide the following general design guidelines applicable to the different sensing tasks.

\section{Substrate materials}

The wearable substrate material used to fabricate the glove is the support of sensors, wires and other electronic components and accessories. Then, the substrate material must provide the required challenging performances of wearability (including adhesion to skin, flexibility, and lightness), reliability, suitability for washing, and physical/chemical stability. The main part of mentioned glove-based systems are made with Lycra, ${ }^{13,8,10}$ nylon or other polymers which provides most of desired mechanical performances of technical fabrics, including electric insulation. For specific requirements (as flameretardant applications), aramidic fibers as Nomex are used. However, some design layouts include fabrics able to conduct electricity, ${ }^{25}$ also called electro-textiles (or e-textiles): they are fabrics with electrically conductive fibers made with metals like copper, silver, etc. with very low electrical resistivity along one or two directions. Local coating of conductive patterns with polymers can be performed to provide the required protection against repeated stretching or abrasion and dedicated test benches are used for accelerated reliability tests. ${ }^{26}$

In more recent prototypes, the flexible PCB technology is used. ${ }^{14}$ Here, the hand back side is covered with 8-layers of flexible PCB with $202.5 \times 124.9 \mathrm{~mm}(0.45 \mathrm{~mm}$ thickness $)$ overall circuit size. Figure 5 shows the PCB glove with all sensors. The flexible PCB substrate is subjected to reliability issues especially in the fingers area due to repeated mechanical loadings. For this reason, the design proposed in Flynn ${ }^{14}$ includes meander-shaped PCB over finger joints: the increased flexibility provided by this geometry reduces internal stress and increases the substrate lifetime. The maximum bending radius of the substrate is also increased, by fitting the angular motion range of human fingers. Discrete sensors like accelerometers and bending/ force sensors are installed on dedicated islands on the PCB substrate. Finally, the development of recent technologies in the field of flexible batteries and printable conductive is opening to the integration of these components to glove-based systems.

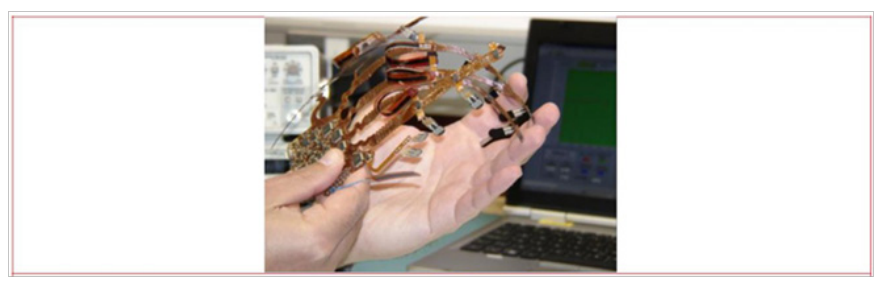

Figure 5 PCB glove under flexion. ${ }^{14}$

\section{Sensors}

Sensors typologies, positioning and arrangement are the main design parameters concerning data acquisition. Sturman and Zeltzer ${ }^{27}$ proposed a theoretical approach for addressing the problem of choosing the most appropriate number and locations of sensors for glove devices. It was based on the analysis of the DoFs of the application and their match to the DoFs of the device. Alternatively, empirical approach can be used as in the case studies described in the following.

\section{Sensors typologies}

The most important parameters in hand rehabilitation measurements are finger joints angles. This is the case of trainings of adduction (movement of fingers toward the hand center) and abduction (movement of fingers away from the hand). The bending sensors normally used for this purpose belong to the following four categories:

a. Piezoelectric films. ${ }^{28}$ Piezoelectric films are well matched to the requirements of e-textiles and wearable devices, by offering low power consumption, wide shape freedom, and suitability to different components. Piezoelectric materials respond to almost any type and magnitude of physical stimuli. Typical electrical outputs are characterized by high voltage and low currents.

b. Optical fibers. They are characterized by lightweight, flexibility and immunity to electro-magnetic interferences, in addition to high sensitivity and reliability. Then they are well suited for implementation in advanced wearable interfaces. ${ }^{21,22,29,30}$ Sensors based on optical fibers are basically represented by waveguides placed on fingers: due to finger bending, stresses are induced into the waveguides due to the differences on mechanical properties of fiber and substrate, resulting in light attenuation. Therefore, the intensity of optical signals can be correlated to the angular displacements of finger joints. Fiber optics sensors used in 5DT Data Glove. ${ }^{31}$

c. Resistive transducers. The internal flexible structure covered with carbon-ink measures the resistance variations versus the bending radius of the transducer (Figure 6) ${ }^{32}$ Resistive transducers are flexible, low cost and easy to adapt in length, and then they could span one single finger joint. In the properties of a flexible resistive sensor are investigated; it is made of conductive polymer thin films deposited on flexible substrates of polyimide. ${ }^{33}$ In many cases, $3,6,8,10,13,14,23,34$ polyester over-laminated bend sensors are also used, as by Flexpoint. ${ }^{35}$ 
d. Hall-effect transducers. These sensors are based on the variation of output voltage in response to a magnetic field. In presence of given magnetic field, specific combination of Hall-effect sensors is able to quantify joint angles. Hall-effect transducers are used, for instance, in the Humanglove. ${ }^{5,36}$

Forces exerted by finger on objects or on another finger are also important parameter to be measured during clinical trainings or medical applications. Forces can be measured at fingertips or on the internal hand surface by means of the sensor typologies reported in the following:

i. Resistive force sensors (FSR, Figure 7). They are simply passive sensors with decreasing resistance in presence of increasing force. This kind of sensors provided by Interlink Electronics ${ }^{37}$ have been applied, ${ }^{14,15}$ while those ones made by Tekscan ${ }^{38}$ have been used in the FuncAssess Glove (Flexiforce). ${ }^{23}$

ii. Piezoresistive fabrics. The piezoresistive effect is the working principle of this transducer, where the electrical resistance of the base material varies under mechanical pressure. This kind of sensor was used, ${ }^{19}$ where it is based on piezoresistive, stretchable knitted fabric ( $72 \%$ nylon, $28 \%$ spandex) and is manufactured by Eeonyx. ${ }^{39}$ The individual fibers within the fabric are coated in the nanoscale with inherently-conductive polymers.

iii. Capacitive pressure sensors. This highly sensitivity pressure sensor, made e.g. by FingerTPS ${ }^{17}$ is used in ${ }^{16}$ to reliably quantify forces applied by the hand. The system has six comfortable capacitive sensors per hand, wirelessly connected to the computer. The sensors have a data rate of $40 \mathrm{~Hz}$ and the full scale range is 10$50 \mathrm{lbs}$ (sensitivity is $0.01 \mathrm{lbs}$ ). Video images are synchronized with tactile data and can be captured and displayed in real time.

iv. Electromyographic (EMG) sensor. Electromyographic signals of forearm muscles can be used also to estimate fingers' forces as demonstrated. ${ }^{16}$

v. Magnetic, angular rate and gravity (MARG) sensors. This setup, in combination with dedicated kinematic model, is used ${ }^{24}$ to estimate force exerted between the hand and deformable objects and the relative position between fingertips and palm. Inertial sensors are used to detect contact events of fingertips against objects thanks to their sensitivity to impact. Once the contact event is detected, the position of the corresponding fingertip is considered as the object reference. Magnetic sensors provide the relative position between fingers and hand surface.

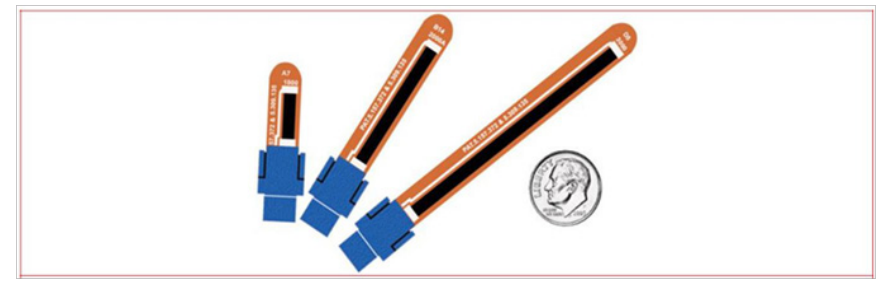

Figure 6 Resistive bend sensors. ${ }^{32}$

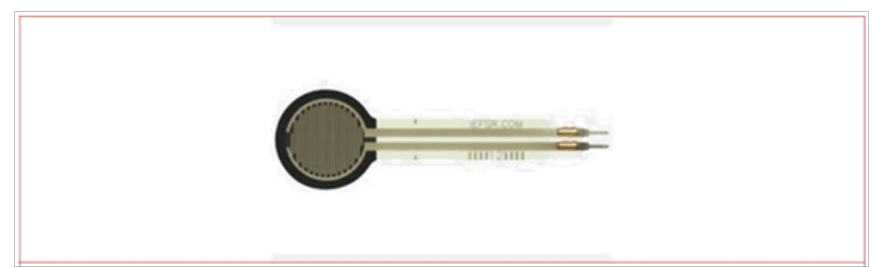

Figure 7 Resistive force sensor (FSR). ${ }^{37}$
Hand position and orientation are crucial for trainings addressed to restore mobility of patients. The hand/arm motion trajectory, for instance, provides significant information about muscles control and body awareness processes. Several strategies, described in the following, were used to evaluate hand position and orientation in space.

a. Inertial measurement units (IMU) and motion processing units (MPU). The combination of three-axes accelerometers and threeaxes gyroscopes is capable of tracking the hand position and orientation. This method is applied ${ }^{21}$ where the 6-axes MEMS inertial sensor is used as motion tracking device ${ }^{40}$ to provide linear and angular acceleration with adjustable scale ranges. The glove described in (Pascual-Leone 2012) integrates the IMU on the hand back side to assess its orientation (roll, pitch, and yaw). The system has also a resting base for the hands and forearms used to reset zero position during training and before each block of trials, via a Hall-effect sensor. The IMU used in this system has 9degrees of freedom provided by three-axial accelerometer, gyroscope, and magnetometer and a microcontroller for computing orientation.

b. Inclinometers and bending sensors. The glove ${ }^{14}$ integrates 16 three-axial accelerometers, one per finger's phalanx and on hand palm, which are used to measure angular orientations (then working as inclinometers). Since the accuracy and resolution of these inclinometers respond to requirements only in quasi-static condition, bending sensors and Kalman filter are also used to provide more precise finger's flexion estimation. Accelerometers also provide additional data about absolute rotation and shaking/ vibration motions.

c. Inertial and magnetic measurement systems (IMMS) through Kalman filtering. Inertial and magnetic sensors are suitable to measure accurately body segments orientation without external actuators or cameras. The data glove described ${ }^{18}$ is based on this setup, coupled with Kalman filter to assess 3D hand and finger kinematics. Multiple extended Kalman filters (EKF) are designed to estimate the optimal orientation trajectories of hand and fingers.

\section{Sensors positioning and arrangement}

After selection of sensors typologies, their positioning and arrangement (and hence number) is the next design strategy step. Sometimes small differences in spatial configuration of sensing elements can lead to huge modifications of the behavior of the whole system. In the following, several solutions proposed in the literature to define sensors architectures are described:

i. Glove-based devices presented ${ }^{3,10,13,23}$ include dedicated pockets where sensors are hosted. In Gentner and Classen, ${ }^{10}$ more specifically, sensors are applied symmetrically over finger joints with this strategy, and the proximal ends are fixed to fabric with medical tape. Depending on fingers length, the distal end of one sensor can overlap the proximal end of another sensor. To avoid contact between sensors placed on fingers, three layers of polyamide/Lycra fabric are sewn on the glove fabric, thereby forming separate pockets where sensors are hosted. ${ }^{3}$ In bending sensors are coupled to pockets able to run up the back of each finger, by allowing each sensor sliding on the same fingers and accommodating length changes during flexion movements. During extension, pockets prevent sensors buckling on fingers. ${ }^{13}$

ii. The configuration of the Shadow Glove Monitor ${ }^{8}$ is significantly different from other examples of measurement gloves because it does not encase fingers with individual pockets. The glove is composed of individual Lycra sleeves for each joint that must 
be monitored, which contains one bend sensor encased in thin plastic sheath. The sensor, plastic sheath, and outer sleeve are secured together at the proximal end of the assembly. The outer sleeve is attached to the dorsal side of the finger by using double sided medical grade adhesive tape. The tape is applied on the skin proximal and distal to the joint such that the joint is free to move.

iii. Other kinds of gloves do not have sleeves but adopt valid solution to fulfill different hand dimensions. The sensors present,,$^{15}$ for instance, are attached to the glove by providing position adjustment according to user requirements. Instead, sensors are represented by special fabric working as tactile detector and, then, are disposed on the whole hand surface extension. ${ }^{19}$

iv. The system contains multiple flexible PCB strings mounted on the back side of hand, fingers and thumb (Figure 8). ${ }^{18}$ Each string deploys three three-axial gyroscope and accelerometer (ST LSM330DLC) pairs, one for each finger or thumb segment. In addition, a three-axial magnetometer (Honeywell HMC5983) is placed on the fingers tip and on the hand back side.

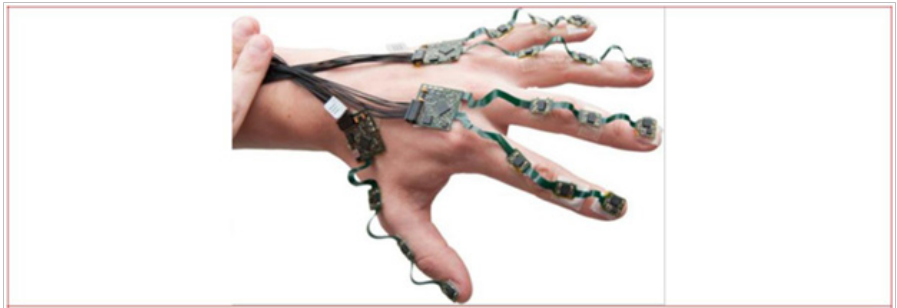

Figure 8 Glove arrangements with flexible PCB. ${ }^{18}$

\section{Validation tests}

Sensors functionality and performances required validation through dedicated tests. Only rarely performance of the overall glove system (substrate, sensors, and electronics) in terms of repeatability and accuracy has been documented in literature due to lack of standard methodologies of validation. Laboratory tests on single sensors have been described, as for piezoelectric bending sensors and force sensors applied to glove-based systems.

The shaking tests are used ${ }^{41,42}$ where the transducer in the cantilever configuration is loaded cyclically with electromechanical shaker, and the electric output is measured. The goal of this investigation is to identify the optimum resistive load that maximizes the generated power. Another laboratory test has been described, with the goal to measure the impact energy of a small mass dropping from $70 \mathrm{~mm}$ height onto the piezoelectric materials fixed to a rigid structure. ${ }^{41}$ The importance of sensor positioning when bending is not uniform along the transducer is emphasized, ${ }^{34}$ with reference to the case of finger bending. Indeed, a longitudinal displacement of few millimeters with respect to the finger joint modifies the bending-voltage curve of the sensor. This definitely represents a drawback when trying to obtain repeatable measurements, depending on the initial sensor position.

Typical documented effect associated to bending sensors is timevarying creep when held in a fixed, bent position. Also, it is sometimes appreciated inaccuracy in returning to the same baseline resistance value (i.e. flat position). For these reasons $\mathrm{s}^{3,8}$ performed "time-varying creep" and "returning" experiments to compare the performances of different commercial bending sensors. Stability evaluation test is performed ${ }^{23}$ to determine sensor drift (i.e. output changes over time) under constant conditions. The drift reveals the propensity of the sensor to undesired alterations of measurement due to changes in actuation angles (bending sensors) or applied loads (force sensors). Finally, the insensitivity of bending sensors readings to variations of squeeze pressures and wrist positioning has been demonstrated. ${ }^{6}$

In general, the overall measurement performance is mostly influenced by substrate flexibility and connection strategy with sensors. This last point represents the main open challenge for wearable sensing systems integrating electronic components or discrete sensors. The development of technologies related to conductive inks and printable circuits are introducing promising alternatives to the integration of traditional electronic elements on clothes; however these methodologies are still not mature and unable to cover all the requirements in terms of transduction and reliability.

\section{Calibration}

Anthropometric dimensions have significant differences among population, including hand size, fingers length and thickness. Then, glove-based systems must fit with different finger locations for different users, which may affect functional measurements. Many gloves require time-consuming and tedious calibration for every user to reduce inaccuracy of sensing performances. Calibration is normally performed by asking the user to place his hands in specified configurations. Also gain and offset parameters must be determined for each sensor to match the global system response between physical motion of hands and sensors readings. Some of the most frequent calibration procedures are described below.

Glove system ${ }^{10}$ integrates bend sensors with linear output. For this transducer typology, only two angle-voltage detections are needed (corresponding to flat position and maximum bent configuration of hand) to calibrate the device. The calibration of overall 14 sensors is performed in about $10 \mathrm{~s}$. Instead, when the transducer output is nonlinear, ${ }^{8}$ the calibration process is pretty longer (approximately $8 \mathrm{~min}$ per person in this case). Two different calibrations are needed: ${ }^{13}$ one for the bending measurement and another for $3 \mathrm{D}$ hand orientation. The first one consists of placing each subject's hand on reference position templates for static angle measurements. The templates indicate the reference for metacarpophalangeal (MCP) and proximal interphalangeal (PIP) joints positions of fingers, which are detected by using a manual goniometer. The IMU calibration test is required for hand orientation in space, and consists in moving the wrist and/ or forearm/arm in six linear and angular directions: roll (supination/ pronation), pitch (wrist extension/flexion), yaw (radial/ulnar deviation), medial-lateral (x), forward-backward (y), and up-down movements (z).

Non-linear output for bending sensors is documented, ${ }^{23}$ where the voltage outputs are measured at finger joints angles of $0^{\circ}, 20^{\circ}, 40^{\circ}, 60^{\circ}$ and $90^{\circ}$. Due to the nonlinear relationship between the voltage outputs and bending angles, the MATLAB shape-preserving interpolation function "pchip" was used. Force sensors with linear output also present in the same device were calibrated by fixing them on a flat surface and applying variable loading masses from 10 to $350 \mathrm{~g}$. Calibration software was used ${ }^{11}$ to set the personal configuration setting of each user/doctor. The default calibration is stored in the Virtual Hand software and then used to control the data glove performances. Additional calibration is required to improve accuracy 
for each sensor. As other gloves, calibration is achieved by completing a set of finger positions. Advanced calibration can be performed by adjusting offset and gain values for each sensor.

The manual and boring calibration practice was been replaces in some cases by alternative strategies. The CyberGlove $\mathrm{III}^{11}$ includes a conversion system, wherein the relationship between the sensors output voltages and finger bending angles is pre-measured by using automated instrument based on dummy finger part. These measurements are stored in a lookup table, which enables to convert by software the output voltages coming from sensor to angles. The advantage of this system is that the glove does not require calibration before each measurement session, which saves a significant amount of time. The Tyndall/UU glove ${ }^{14}$ is controlled by the combination of sensors and bespoke software which does not require calibration for each user. The simultaneous use of finger joint motions by means of multiple accelerometers, bending sensors and force sensors eliminates the need for calibration. In fact, accelerometers improve accuracy of both static and dynamic finger joint detections. Bending sensors work in collaboration with accelerometers to accurately measure finger joint angles, velocity and acceleration. All data are sent to the software that filters different information and returns the exact hand spatial positioning independently from the user's hand size.

\section{Signal processing}

The conditioning circuit is addressed to enhancement of the signal coming from sensors by using appropriate filtering techniques. Sensors output are still raw and therefore require specific electronic processing before being available to be processed by a microcontroller. An example of sensor enhancement is explained, ${ }^{3}$ where simple circuit is used to increase sensor resolution by amplifying its dynamic range. The output response of bending sensors in the range $0-120^{\circ}$ is globally nonlinear: after the first almost-linear region of the characteristic curve (approximately in the range $0-100^{\circ}$ ), a saturation region is present, not suitable for measurements. Then, the shape of the sensor curve can be modified to increase the sensor dynamic range, and thereby to enhance the sensor resolution, by simply modifying the sensor signal conditioning circuit.

Similarly in a signal conditioning circuit made only of resistors and operational amplifiers has been used to obtain a linear relationship between the sensor resistance and the bending angle. ${ }^{10}$ The idea was to place a resistor parallel to the sensor and then connect the sensor to the amplifier. Finally, the conditioning circuit ${ }^{23}$ includes simple currentto-voltage converter. In Figure 9 the signal conditioning circuits of one bend sensor and one force sensor are shown.

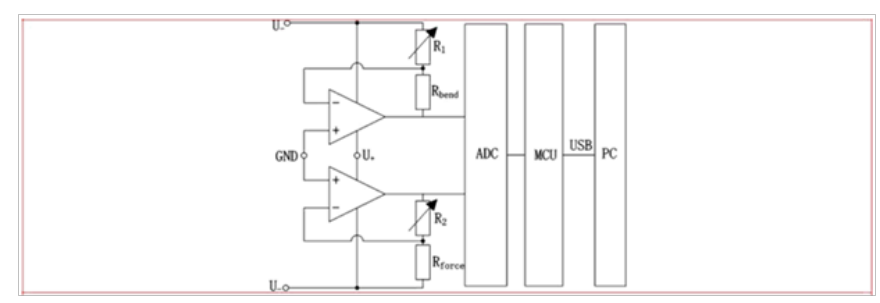

Figure 9 Signal conditioning circuits of bend sensor (Rbend) and force sensor (Rforce). ${ }^{23}$

After conditioning circuit, the analog-to-digital (ADC) signal conversion is performed. ${ }^{43}$ Some of the most used ADCs mentioned in literature are reported in Table 1 with reference to their properties.
The digital output coming from the ADC is finally sent to the microcontroller by different communication protocols. The most used protocols are SPI (serial peripheral interface), I2C (inter integrated circuit) and UART (universal asynchronous receiver-transmitter). The most used microcontroller units are reported in Table 2.

Table I Most common ADC used in literature for glove-bases systems

\begin{tabular}{lll}
\hline Typology & Properties & Reference \\
\hline I2 bit ADC & $/$ & $\begin{array}{l}\text { Simone et al. } \\
\text { Humanglove }\end{array}$ \\
$\begin{array}{l}\text { Analog Devices } 125 \mathrm{kS} / \mathrm{s} \\
\text { ADC }\end{array}$ & $\begin{array}{l}2.7 \mathrm{~V} \text { to } 5.25 \mathrm{~V} \text {, micro } \\
\text { power, } 8 \text { channels }\end{array}$ & Analog Devices ${ }^{43}$ \\
ADC camera & $/$ & De Pasquale et al..$^{42}$ \\
NI-6008 I0kS/s ADC & 12 bit, 8 channels & Oess et al. ${ }^{3}$ \\
$\begin{array}{l}\text { National Semiconductor } \\
\begin{array}{l}\text { Corporation 0808/0809 } \\
\text { ADC }\end{array}\end{array}$ & $\begin{array}{l}8 \text { bit, } 8 \text { channels } \\
\text { multiplexer }\end{array}$ & Singh et al. ${ }^{15}$ \\
$\begin{array}{l}\text { Analog Devices AD7490 } \\
\text { ADC }\end{array}$ & 12 bit, 16 channels & Zheng et al. ${ }^{23}$ \\
\hline
\end{tabular}

Table 2 Most common microcontroller units used in literature for glovebases systems

\begin{tabular}{ll}
\hline Typology & Reference \\
\hline Motorola HC I2 & Edmison et al. ${ }^{28}$ \\
Microchip PIC & $\begin{array}{l}\text { Tavares, } \text { Abreu et al. }{ }^{21} \text {; Tavares, Souse et al. } .^{22} \text {; } \\
\text { Saggio et al. }{ }^{44}\end{array}$ \\
Atmel ATmega328 & De Pasquale et al. ${ }^{42}$ \\
Texas Instruments MSP430 & Simone et al. ${ }^{8}$ Z Zheng et al..$^{23}$ \\
Philips P89v5IRD2 & Singh et al. ${ }^{15}$ \\
Atmel XMEGA & Kortier et al. ${ }^{18}$ \\
Atmel ATmega I28I low & Flynn et al. ${ }^{14}$ \\
power
\end{tabular}

\section{Connectivity and graphical interface}

\section{Connectivity}

The microcontroller unit is connected to the PC for programming and/or data management. The connection is basically wired or wireless. For glove-based applications, the wireless solution is preferred, despite power consumption significantly increases. Wired connection through USB (universal serial bus) cable has been chosen. ${ }^{18,21,23}$ Bluetooth wireless connection is used by Humanglove ${ }^{36}$ and 5DT Data Glove ${ }^{31}$ (up to a range of $20 \mathrm{~m}$ for 8 hours autonomy with a single battery pack). Both Bluetooth and USB cable are used. ${ }^{21}$ Another typology of wireless connection is provided by transceivers: for instance, the MRF24J40MA transceiver supporting the ZigBee protocol for wireless personal area network (WPAN) is used. ${ }^{33}$ Here, with continuous monitoring activity, the expected battery lifetime is 3 days. A $250 \mathrm{~kb} / \mathrm{s}$ Chipcon Wireless Transceiver $(2.4 \mathrm{GHz}$ IEEE 802.15.4) is used. ${ }^{44}$ The CC2420 Texas Instrument single chip (2.4GHz, IEEE 802.15.4 compliant) transceiver is applied, ${ }^{26}$ which is designed for low-power and low-voltage wireless applications from. 


\section{Graphical interface}

The graphical user interface is the last step in designing and prototyping glove-based medical systems. This module provides the final user (doctor, rehabilitative trainer, patient, etc.) a friendly interface that communicates data with immediateness. Without particular graphical interfaces, standard software for data elaboration can be used. For instance, sensors described ${ }^{3}$ were sampled continuously at $100 \mathrm{~Hz}$ by using LabVIEW, MATLAB scripts are used to receive, display and save data. ${ }^{18,23}$ Software for the arm motion sampling written in $\mathrm{NesC}$ (an extension to the $\mathrm{C}$ language) and running on TinyOS operating system is applied. ${ }^{8}$

The adoption of graphical user interface (GUI $)^{44}$ provides animations and visual information for non-specialist users able to facilitate the proper use of the whole system. The GUI has been obtained in $\mathrm{C}++$ language, by means of Windows application program interfaces (API) and DirectX 9.0c. Then hand movements are recorded by the data glove and are real-time reproduced via avatar in virtual 3D environment with Blender software. In Dipietro et al. ${ }^{5}$ data acquisition is performed through a proprietary software package called Graphical Virtual Hand. This program calibrates the glove and displays virtual hand that mirrors the movements of the user's hand. Present a gamelike interface. ${ }^{20-22} \mathrm{~A}$ graphical application has been developed in order to monitor each finger displacement and to implement rehabilitation exercises. This GUI uses the microchip graphics library functions and has been developed with the graphics display designer GDDX. Besides a functional virtual environment interfaces with the glove and IMU (inertial measuring unit), it provides faithful virtual representation of the user's hand (Figure 10).

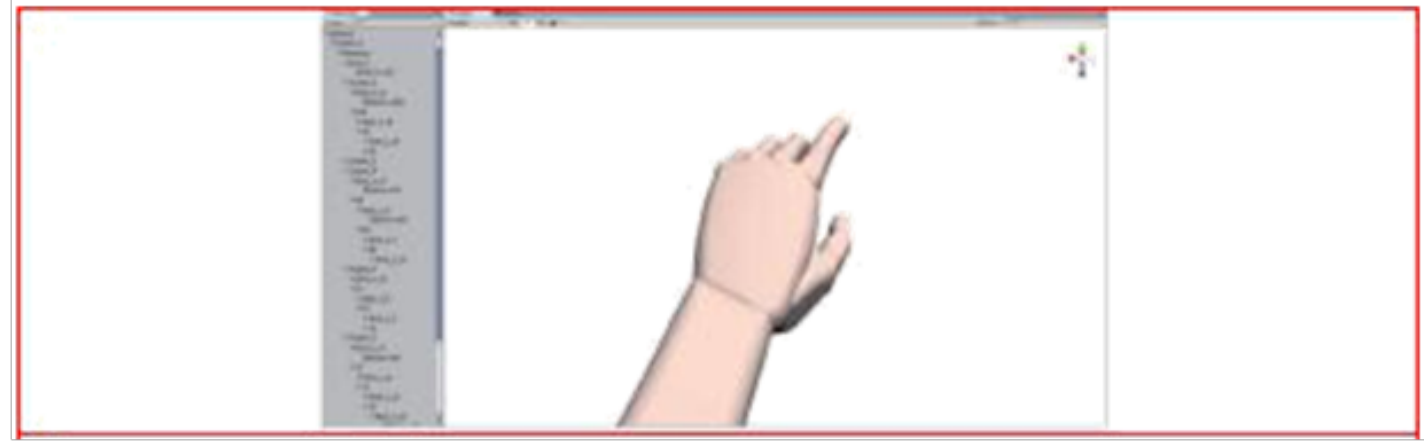

Figure $103 \mathrm{D}$ model of the hand in the virtual environment. ${ }^{21,22}$

\section{Power management and harvesting issues}

The power management unit (PMU) is responsible of the supply of the whole system. Gloves connected by wire to the PC receive the necessary power supply by the cable, while gloves with wireless connection usually include portable PMU in their packaging. First requirement for PMUs is the small weight and size. The Shadow Glove Monitor, ${ }^{8}$ for instance, has 2AA batteries, suitable for at least $24 \mathrm{~h}$ of sensing mode supplying. The wearable system weigh is approximately $145 \mathrm{~g}$ (including five sensors and sleeves: $5 \mathrm{~g}$, signal conditioning box: $85 \mathrm{~g}$, two AA batteries: $55 \mathrm{~g}$ ). Rechargeable batteries are present ${ }^{2-23}$ where the embedded electronic circuit is able to manage the charging process by USB connection.

In latest laboratory tests, the energy harvesting strategy has been used to increase the battery lifetime before discharge. The kinetic energy of hands has been converted to electric power to supply the glove-based system. For instance, GoldFinger glove ${ }^{42}$ makes use of piezoelectric fiber composites (PFCs, Figure 11) with high flexibility. Monolithic piezoelectric ceramics are rigid and heavy, than not suitable for wearable applications. The PFCs consist of unidirectional aligned ceramic fibers (diameter range: $10-250 \mu \mathrm{m}$ ) embedded in an epoxy matrix and sandwiched between two copper clad polymeric laminates. PFCs produce approximately five times the voltage in comparison to polymer films. ${ }^{41}$ In De Pasquale et al. ${ }^{42}$ the circuit able to harvest piezoelectric energy is presented. The voltage generated by the energy harvester has a random shape. For this reason a diode bridge is introduced. It provides first voltage rectification by means of leveling capacitor. Then, operational controls regulate the charge/ discharge of internal capacitance by allowing the current to flow towards the battery only when charge voltage threshold is reached. In conclusion, flexible materials provide non-invasive power harvesting medium able to collect energy lost in bending, stretching, and impact movements of the hand.

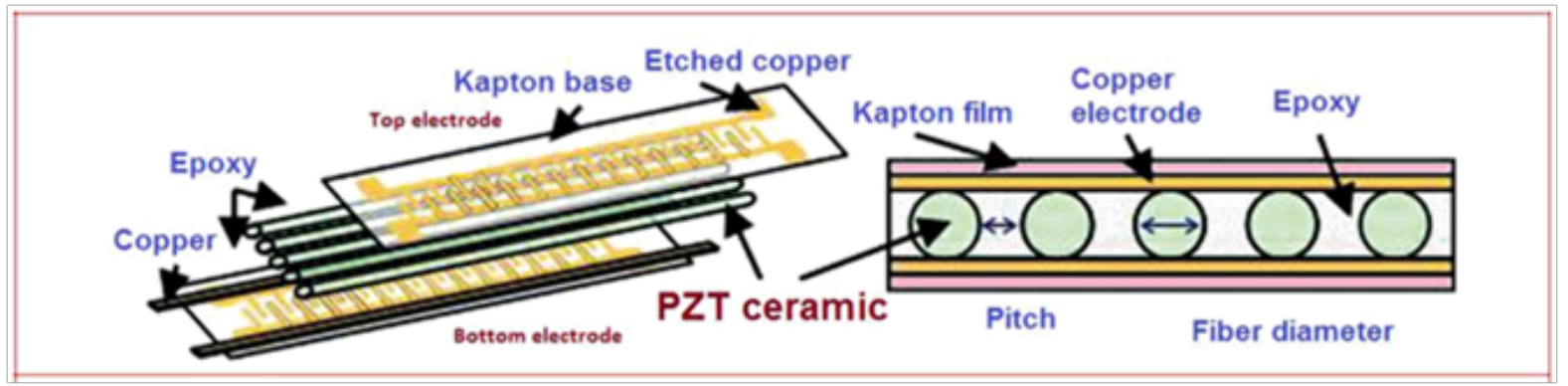

Figure I I Piezoelectric fiber composite. 


\section{Conclusion}

Sensor gloves have been used in a variety of applications, such as robotics and virtual reality, but over the last decades medical purpose has gained a lot of centrality. This paper has firstly emphasized that the importance of research in glove devices has grown up, especially as far as the medicine and health care are concerned. The roadmap for designing and prototyping glove-based system for clinical assessments is provided by this paper by considering the most important experiences documented in this field and their fundamental characteristics. Although sensor gloves have large applicability in hand function assessment, their realistic clinical use is still limited by some issues: size and weight, wearability, wireless connection, packaging, interfacing strategies, and cost. In-progress researches in the field of wearable sensing gloves ${ }^{45}$ are addressed to overcome these limitations. Very promising opportunities for high-potential devices are evident in motion rehabilitation of hand and, in general, in health care.

\section{Acknowledgements}

None.

\section{Conflict of interest}

Author declares there is no conflict of interest in publishing the article.

\section{References}

1. Dipietro L, Sabatini AM, Member S, et al. A survey of glovebased systems and their applications. IEEE T Syst Man Cy C. 2008;38(4):461-82.

2. Carmeli E, Patish H, Coleman R. The aging hand. $J$ Gerontol A Biol Sci Med Sci. 2003;58(2):146-52.

3. Oess NP, Wanek J, Curt A. Design and evaluation of a low-cost instrumented glove for hand funcion assessment. J Neuroeng Rehabil. 2012;9:2.

4. Zimmerman T. Optical flex sensor. US Patent 4542 291. 1982.

5. Dipietro L, Sabatini AM, Dario P. Evaluation of an instrumented glove for hand-movement acquisition. $J$ Rehabil Res Dev. 2003;40(2):179-89.

6. Williams NW, Penrose JM, Caddy CM, et al. A goniometric glove for clinical hand assessment: construction, calibration and validation. J Hand Surg Am. 2000;25(2):200-7.

7. Williams NW. The virtual hand. J Hand Surg. 1997;22B(5):560 67.

8. Simone LK, Sundarrajan N, Luo X, et al. A low cost instrumented glove for extended monitoring and functional hand assessment. $J$ Neurosci Methods. 2007;160(2):335-348.

9. Swallow L, Siores E. Tremor suppression using smart textile fibre systems. Text Bioeng Informatics Symp Proc. 2009;1(4):142-46.

10. Gentner R, Classen J. Development and evaluation of a lowcost sensor glove for assessment of human finger movements in neurophysiological settings. $J$ Neurosci Methods. 2009;178(1):138-47.
11. CyberGlove III.

\section{X-IST Data Glove.}

13. Pascual-Leone A. Development of a low-cost virtual realitybased smart glove for rehabilitation. Int Conf Disabil Virtual Real \& Assoc Technol. 2012;279-86.

14. O'Flynn B, Torres Sanchez J, Connolly J, et al. Novel smart sensor glove for arthritis rehabiliation. 2013;1-6.

15. Singh G, Boddu S, Chakravorty I, et al. An instrumented glove for monitoring force during object manipulation. IEEE Point-of-Care Healthc Technol. 2013;16-18.

16. Ju Z, Liu H. Human hand motion analysis with multisensory information. IEEE/ASME Trans Mechatronics. 2014;19(2):45666.

17. Finger TPS. Finger TPS Pressure Sensor System.

18. Kortier HG, Sluiter VI, Roetenberg D, et al. Assessment of hand kinematics using inertial and magnetic sensors. J Neuroeng Rehabil. 2014;11(70):1-14.

19. Büscher GH, Kõiva R, Schürmann,C, et al. Flexible and stretchable fabric-based tactile sensor. Rob Auton Syst. 2015;63(3):244-52.

20. Tavares R, Abreu P, Quintas MR. Instrumented glove for rehabilitation exercises." Proc Experiment International Conference. 2015;107-108.

21. Tavares R, Souse PJ, Abreu P, et al. Virtual environment for instrumented glove. Proc REV Intern Conf. 2016;311-12.

22. Tavares R, Abreu P, Quintas MR. Data acquisition glove for hand movement impairment rehabilitation. Int $J$ Online Eng. 2016;12(4):52-54.

23. Zheng Y, Peng Y, Wang G, et al. Development and evaluation of a sensor glove for hand function assessment and preliminary attempts at assessing hand coordination. Meas J Int Meas Confed. 2016;93:1-12.

24. Mohammadi M, Baldi TL, Scheggi S, et al. Fingertip force estimation via inertial and magnetic sensors in deformable object manipulation. IEEE Haptics Symp. 2016;284-89.

25. Pretreatments C, Cotton O. Hauser PJ. Textile News. 2014:3-5.

26. De Pasquale G, Mura A. Accelerated lifetime tests on e-textiles: design and fabrication of multifunctional test bench. $J$ Ind Text. 2017;47(8):1925-43.

27. Sturman DJ, Zeltzer D. A design method for 'whole-hand' humancomputer interaction. ACM Trans Inf Syst. 1993;11(3):219-38.

28. Edmison J, Jones M, Nakad Z. et al. Using piezoelectric materials for wearable electronic textiles. Proc Int Symp Wearable Comput. 2002:41-48.

29. Fujiwara E, Miyatake DY, Ferreira M, et al. Development of a glove-based optical fiber sensor for applications in human-robot interaction. 2013;123-24.

30. Nishiyama M, Watanabe K. Wearable sensing glove with embedded hetero-core fiber-optic nerves for unconstrained hand 
motion capture. IEEE Trans Instrum Meas. 2009;58(12):39954000 .

31. 5DT Data Glove.

32. Tactilus ${ }^{\circledR}$ Flex Sensor.

33. Saggio G. Mechanical model of flex sensors used to sense finger movements. Sensors Actuators A Phys. 2012;185:53-58.

34. Oess NP, Wanek J, Van Hedel HJ. Enhancement of bend sensor properties as applied in a glove for use in neurorehabilitation settings. Proc EMBC Int Conf. 2010;5903-5906.

35. Flexpoint.

36. Humanglove.

37. Interlink Electronics.

38. Tekscan.
39. Eeonyx. Smart fabrics for the internet of things.

40. InvenSense.

41. Swallow LM, Luo JK, Siores E, et al. A piezoelectric fibre composite based energy harvesting device for potential wearable applications. Smart Mater Struct. 2008;17(2):25017.

42. De Pasquale G, Kim SG, De Pasquale D. GoldFinger: Wireless human-machine interface with dedicated software and biomechanical energy harvesting system. IEEE/ASME Trans Mechatronics. 2015;21(1):565-75.

43. Analog Devices.

44. Saggio G, Bocchetti S, Pinto CA, et al. Wireless data glove system developed for HMI. Proc ISABEL Int Conf. 2010;1-4.

45. Mastrototaro L. Design and prototyping of a wearable sensing system for clinical rehabilitation trainings. Torino: Polytechnic University of Turin. 2017. 